CHRONIC OBSTRUCTIVE PULMONARY DISEASE

\title{
Continuous and intermittent exercise responses in individuals with chronic obstructive pulmonary disease
}

\author{
S Sabapathy, R A Kingsley, D A Schneider, L Adams, N R Morris
}

Thorax 2004;59:1026-1031. doi: 10.1136/thx.2004.026617

See end of article for authors' affiliations

Correspondence to: Mr S Sabapathy, School of Physiotherapy and Exercise Science, Gold Coast Campus, Griffith University, PMB 50 Gold Coast Mail Centre, Queensland 9726, Australia; S.Sabapathy@ griffith.edu.au

Received 16 April 2004 Accepted 13 September 2004

\begin{abstract}
Background: While the acute physiological responses to continuous exercise have been well documented in individuals with chronic obstructive pulmonary disease (COPD), no previous study has examined the response to intermittent exercise in these patients.

Methods: We examined the physiological responses of 10 individuals with moderate COPD (forced expiratory volume in 1 second $52(15) \%$ predicted) who performed both an intermittent (1 min exercise and rest intervals) and a continuous cycle ergometer test on separate days. Both intermittent and continuous exercise tests were performed at the same power output, calculated as $70 \%$ of the peak power attained during an incremental exercise test.

Results: Intermittent exercise was associated with significantly lower values for oxygen uptake, carbon dioxide output, expired ventilation, heart rate, plasma lactate concentration, and ratings of breathlessness than continuous exercise. Subjects were able to complete a significantly greater total amount of work during intermittent exercise (71 (32) kJ) than during continuous exercise (31 (24) kJ). The degree of dynamic lung hyperinflation (change in end expiratory lung volume) was significantly lower during intermittent exercise $(0.23(0.07) \mathrm{l})$ than in continuous exercise $(0.52(0.13) \mathrm{l})$.

Conclusions: The greater amount of work performed and lower measured physiological responses achieved with intermittent exercise may allow for greater peripheral training adaptations in individuals with more limited lung function. The results suggest that intermittent exercise may be superior to continuous exercise as a mode of training for patients with COPD.
\end{abstract}

E ndurance training has been shown to reduce ventilatory demand during exercise and improve peripheral muscle function in patients with chronic obstructive pulmonary disease (COPD). ${ }^{12}$ While these results are encouraging, individuals with moderate to severe COPD are often unable to sustain prolonged periods of exercise at intensities that could maximise peripheral muscle and central cardiovascular adaptations. ${ }^{3}$ Consequently, intermittent exercise (IE) has been suggested as an alternative training modality that may be better tolerated in patients with COPD. ${ }^{45}$ Intermittent exercise is characterised by repeated short bouts of exercise separated by periods of rest and may be more suited to COPD patients whose daily activities typically require short bursts of exertion interspersed with periods of recovery. ${ }^{6}$

In healthy individuals IE is associated with lower values for heart rate, oxygen uptake $\left(\dot{\mathrm{V}}_{2}\right)$, expired ventilation $(\dot{\mathrm{V}} \mathrm{E})$, and blood lactate concentration than continuous exercise (CE)..$^{8}$ An important functional outcome is that total work performed is greatly increased during IE compared with CE when performed at the same absolute intensity. ${ }^{9}$ Moreover, Morris and colleagues ${ }^{8}$ showed that the lower $\dot{\mathrm{VO}}_{2}$ response associated with IE compared with CE performed at 70\% of peak power was primarily due to the exponential $\dot{\mathrm{V}}_{2}$ kinetic response typical of a step change in activity level (ontransient $\dot{\mathrm{V}}_{2}$ kinetics). At the onset of constant load exercise a transient exponential increase in $\dot{\mathrm{V}}_{2}$ is observed, attaining a new steady state level within 120-180 seconds during light to moderate intensity exercise. ${ }^{10}$ Even during higher intensity exercise that engenders a slow component of increasing $\dot{\mathrm{V}}_{2}$, the on-transient kinetic response remains exponential. ${ }^{11}$ Thus, for exercise intervals greater than 120 seconds, the $\dot{\mathrm{V}}_{2}$ attained at the end of each exercise interval approaches that reached during $\mathrm{CE}$ at the same exercise intensity. This suggests that the duration of the exercise period is an important factor in determining the IE response, with longer exercise intervals reducing the differences observed between $\mathrm{CE}$ and IE.

To date there have been no studies examining the acute response to IE in individuals with COPD. However, Vogiatzis et $a l^{4}$ reported that the perception of dyspnoea was systematically lower during IE training than during CE training in patients with moderate to severe COPD, despite IE being performed at a greater relative intensity than CE. A reduced ventilatory demand and lower degree of dynamic lung hyperinflation during IE could contribute to a reduction in dyspnoea and an improvement in exercise tolerance, independent of the metabolic demand for this mode of exercise. Studies in patients with COPD have also demonstrated slowed on-transient $\dot{\mathrm{V}}_{2}$ kinetics (measured as the time constant or time taken to attain $63 \%$ of the asymptotic amplitude) compared with healthy control subjects during submaximal exercise. ${ }^{12}{ }^{13}$ Slower kinetics may in turn result in a relatively lower $\dot{\mathrm{V}}_{2}$ amplitude during IE.

Severe deconditioning and dyspnoea with exertion are major deterrents to physical activity in COPD patients and could reduce compliance with exercise rehabilitation programmes. ${ }^{14}$ If total work performed and/or time to exhaustion are increased and perceived intensity of breathlessness reduced during IE compared with $\mathrm{CE}$, then IE may be a useful training mode in the pulmonary rehabilitation setting. The purpose of the present study was therefore to compare the physiological responses to IE using 1 minute exercise and 1 minute rest intervals, and CE performed at the same

Abbreviations: $C E$, continuous exercise; $E E L V$, end expiratory lung volume; $\mathrm{FEV}_{1}$, forced expiratory volume in 1 second; FVC, forced vital capacity; FRC, functional residual capacity; HRpeak, peak exercise heart rate; IC, inspiratory capacity; IE, intermittent exercise; TLCO, carbon monoxide transfer factor; $\mathrm{TLC}$, total lung capacity; $\mathrm{V}_{2}$ peak, peak $\mathrm{O}_{2}$ uptake; VEpeak, peak expired ventilation 
absolute power output in a group of COPD patients with moderate airflow obstruction. Performing IE and CE at the same absolute intensity will normalise the metabolic demand and allow for a comparison of the physiological responses between the two exercise modes. It is hypothesised that (1) exercise tolerance (total work performed) will be greater during IE than during CE, (2) IE will be associated with a lower degree of physiological perturbation $\left(\dot{\mathrm{V}}_{2}, \dot{\mathrm{V}}_{\mathrm{E}}\right.$, blood lactate concentration, dyspnoea), (3) the degree of dynamic hyperinflation will be lower in IE than in $\mathrm{CE}$, and (4) dynamic hyperinflation will be positively correlated with breathlessness.

\section{METHODS}

\section{Subjects and experimental design}

Five men and five women with COPD of mean (SD) age 68 (8) years participated in this study. Inclusion criteria for the study were ( 1 ) patients classified as having moderate COPD, ${ }^{15}$ (2) shortness of breath on exertion, and (3) no documented history of substantial co-morbidity. Subjects visited the laboratory on four separate occasions with each visit separated by at least 48 hours. During the first visit the subjects performed pulmonary function tests, were familiarised with the exercise testing procedures, and provided written informed consent. The second visit was used to determine each subject's maximal exercise capacity. During the subsequent two visits the subjects performed CE and IE on a cycle ergometer. The order of the CE and IE tests was randomised, and the subjects performed only a single bout of exercise on either test day. The experimental protocol was approved by the Griffith University Human Research ethics committee.

\section{Experimental procedures \\ Pulmonary function assessment}

Spirometry, static lung volumes, lung transfer factor (TLCO), and inspiratory capacity (IC) during exercise were measured using a closed circuit pulmonary function testing system (Collins GS Modular PFT, Braintree, MA, USA). Total lung capacity (TLC) was measured using the helium dilution method while TLCo was assessed with the single breath carbon monoxide test.

\section{Determination of peak $\mathrm{VO}_{2}$ for cycling}

The incremental exercise test used to measure peak $\dot{\mathrm{V}}_{2}$ was performed on a Lode cycle ergometer (Excalibur Sport, Groningen, The Netherlands). Subjects commenced unloaded cycling for 3 minutes and then the power output was increased by $4 \mathrm{~W} / 30 \mathrm{~s}$ for men and by $3 \mathrm{~W} / 30 \mathrm{~s}$ for women until volitional termination of the test.

During the incremental cycling test subjects breathed through a mouthpiece and wore a nose clip. $\dot{\mathrm{V}}_{2}$, carbon dioxide output $\left(\dot{\mathrm{V}}_{\mathrm{CO}}\right)$ and $\dot{\mathrm{V}} \mathrm{E}$ were measured breath by breath and averaged over 30 second intervals using a metabolic measuring system (MedGraphics CPX/D, St Paul, MN, USA). A 12-lead electrocardiograph (ECG) configuration was used to monitor cardiac rhythm and to determine heart rate (MedGraphics $\mathrm{CardiO}_{2}$, St Paul, MN, USA). Peak exercise values for incremental cycling were calculated as the average of the two highest consecutive 30 second values obtained before termination of exercise.

\section{Continuous and intermittent exercise tests}

The power output for the continuous and intermittent exercise tests was calculated as $70 \%$ of the power output achieved at peak $\dot{\mathrm{V}}_{2}$. Each of the $\mathrm{CE}$ and IE tests was preceded by 3 minutes of unloaded cycling. The IE test consisted of 1 minute of exercise interspersed with 1 minute of rest-that is, an exercise to rest ratio of $1: 1$. Subjects were encouraged to cycle until the limit of tolerance during both exercise tests. The tests were terminated if subjects were able to complete 30 minutes of CE and/or 60 minutes of IE. Gas exchange indices were measured as described for the incremental exercise test. The breath by breath data were smoothed using a middle five of seven breath averaging procedure. Heart rate and rhythm (Lohmeier M607, Munich, Germany) were monitored during the tests with the ECG electrodes placed in a CM5 configuration. Gas exchange and heart rate values are reported as the peak value attained within a 10 second bin at the end of every minute of exercise. For example, the value at the end of the first minute of exercise for both modes would represent the peak value attained between 0:50 and 1:00 min:s. Total work completed was calculated as the product of exercise time and external power output.

\section{Effect of $\mathrm{VO}_{2}$ kinetics on intermittent exercise response}

In order to determine if any differences in $\dot{\mathrm{V}}_{2}$ between the exercise modes were related to the on-transient kinetics of $\dot{\mathrm{V}}_{\mathrm{O}_{2}}$, the time constant $(\tau)$ of the initial rise in $\dot{\mathrm{V}}_{2}$ (the phase II or primary component) during the CE bout was calculated (see online data supplement at www.thoraxjnl.com/ supplemental). The phase II $\tau(\tau=82$ (8) seconds, $N=7)$ was then used to predict the $\dot{\mathrm{V}}_{2}$ amplitude at 1 minute of exercise and compared with the mean measured $\dot{\mathrm{V}}_{2}$ obtained during the exercise intervals of the IE bout. ${ }^{8}$ To demonstrate the effect of the speed of the on-transient component on the $\dot{\mathrm{V}}_{2}$ amplitude for $\mathrm{IE}$, a $\tau$ value of 42 seconds was also used to predict the IE response. This $\tau$ value was previously determined for healthy older subjects performing constant load exercise at a similar relative intensity to that used in the present study. ${ }^{16}$

\section{Determination of plasma lactate concentration}

Before commencement of the CE and IE tests an indwelling cannula was inserted into an antecubital vein of each subject. The plasma lactate concentration was determined (CibaCorning Blood Gas Analyser, Medfield, MA, USA) from blood samples obtained at rest, at the end of 3 minutes of unloaded cycling, after 3 and 6 minutes of CE and IE, and at the end of the exercise bouts.

Table 1 Subject characteristics, pulmonary function, and peak exercise values obtained during incremental exercise

\begin{tabular}{|c|c|c|}
\hline & Measured & $\%$ predicted \\
\hline Age (years) & $68(8)$ & \\
\hline Height $(\mathrm{cm})$ & $165(12)$ & \\
\hline Body mass $(\mathrm{kg})$ & $68.7(14.0)$ & \\
\hline \multicolumn{3}{|l|}{ Pulmonary function } \\
\hline $\mathrm{FEV}_{1}$ (I) & $1.35(0.50)$ & $52(15)$ \\
\hline FVC (I) & $2.86(1.15)$ & $87(8)$ \\
\hline $\mathrm{FEV}_{1} / \mathrm{FVC}(\%)$ & $48(7)$ & \\
\hline TLC (I) & $6.64(1.87)$ & $126(18)$ \\
\hline FRC (I) & $4.40(1.21)$ & $144(20)$ \\
\hline TLCO $(\mathrm{ml} / \mathrm{min} / \mathrm{mmHg})$ & $9.32(0.76)$ & $46(13)$ \\
\hline \multicolumn{3}{|l|}{ Incremental exercise test } \\
\hline $\mathrm{VO}_{2}$ peak $(\mathrm{ml} / \mathrm{kg} / \mathrm{min})$ & $14.8(2.3)$ & $61.3(14.5)$ \\
\hline Peak power (W) & $57(25)$ & \\
\hline Vepeak (I/min) & $38.4(15.3)$ & $82.0(8.4)$ \\
\hline HR peak (beats/min) & $126(13)$ & $82.6(9.6)$ \\
\hline
\end{tabular}

Values presented are mean (SD).

FEV 1 , forced expiratory volume in 1 second; FVC, forced vital capacity; TLC, total lung capacity; FRC, functional residual capacity; TLCO, carbon monoxide transfer factor; $\mathrm{V}_{2}$ peak, peak $\mathrm{O}_{2}$ uptake; VEpeak, peak expired ventilation; HRpeak, peak exercise heart rate. 

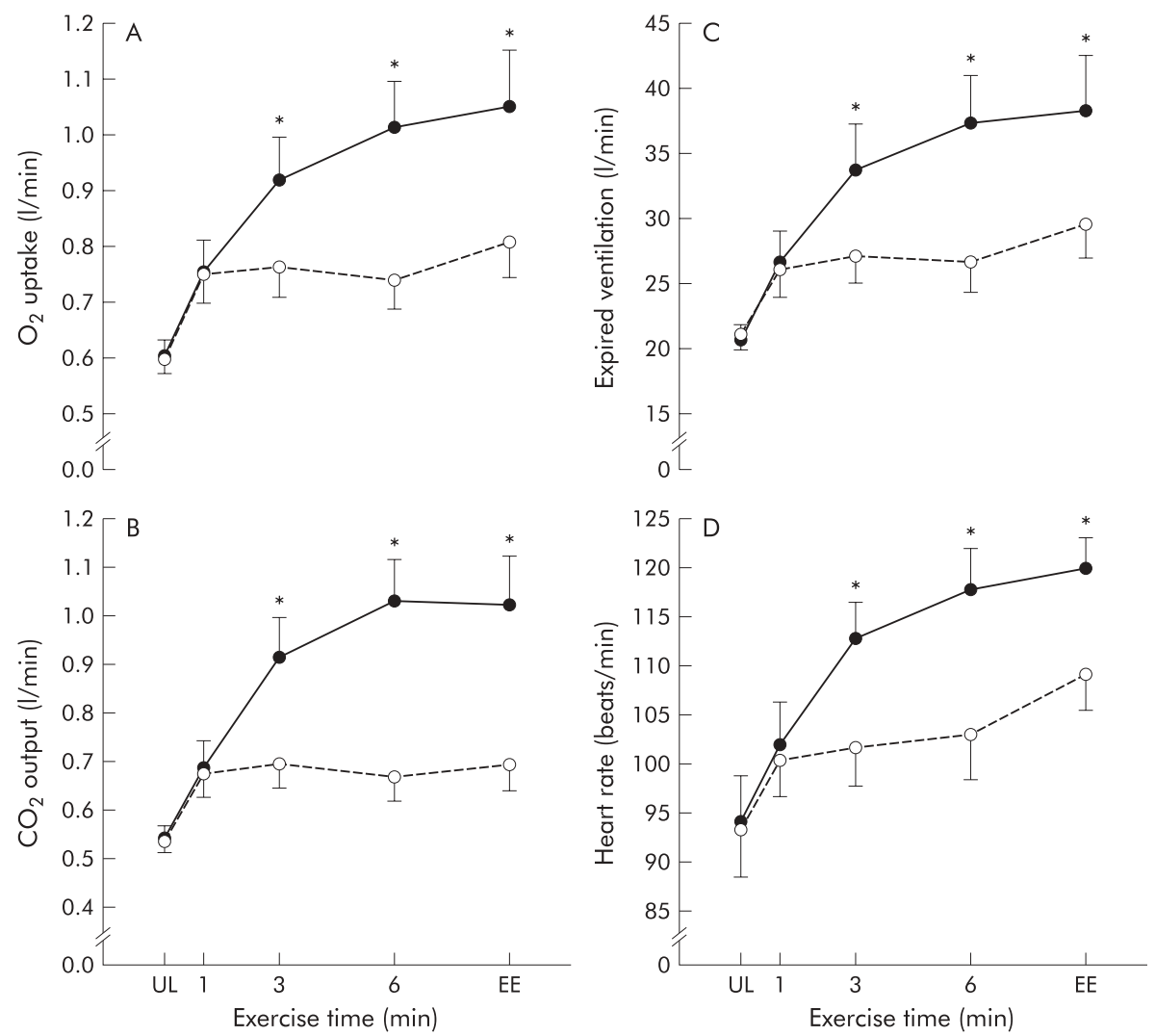

Figure 1 (A) Oxygen uptake, (B) $\mathrm{CO}_{2}$ output, (C) expired ventilation and (D) heart rate responses during continuous (closed circles) and intermittent exercise (open circles). Error bars denote SE. The rest intervals were eliminated from the IE trend to equalise the timeline between exercise modes. UL: unloaded cycling; EE: end exercise. ${ }^{*} p<0.05$, continuous exercise $v$ intermittent exercise.

Measurement of inspiratory capacity and end expiratory lung volume

End expiratory lung volume (EELV) was calculated by subtracting IC from the TLC. Inspiratory capacity was measured at rest (while seated on the cycle ergometer), following 7 minutes of exercise, and at the end of CE and IE. The IC manoeuvre was demonstrated before each test by one of the investigators. At each measurement point the subjects were prompted and encouraged to inspire maximally to TLC. The sampling lines to the pulmonary function system and the pneumotachograph and gas sampling lines to the metabolic cart were interfaced through a single mouthpiece worn throughout the duration of the CE and IE tests.

\section{Ratings of breathlessness}

During the CE and IE tests subjects were asked to provide ratings of their perceived shortness of breath using a word labelled visual analogue scale. ${ }^{17}$ Following a clear explanation of the scale, the subjects were asked to rate their breathlessness at rest, following 7 minutes of exercise, and at the end of exercise.

\section{Statistical analysis}

All results are presented as mean (SE) unless otherwise stated. A two way analysis of variance was performed to detect changes in the dependent variables across the two within subject factors (time and exercise mode). Post hoc tests with Bonferroni adjustments were used when a significant interaction or main effect was identified. The differences in time to exhaustion, total work completed, EELV, and subjects' rating of breathlessness between the two exercise modes were compared using dependent samples $t$ tests. The relationship between EELV and perceived intensity of breathlessness was assessed using Pearson's correlation coefficient. Statistical significance was accepted at $\mathrm{p}<0.05$. Data were analysed using SPSS Version 11.5 (Chicago, IL, USA).

\section{RESULTS}

The physical characteristics of the subjects, pulmonary function test results, and peak exercise values obtained during incremental cycling are shown in table 1 . The pulmonary function results showed that the subjects had moderate COPD. Peak $\dot{\mathrm{V}}_{2}$ was reduced by about $40 \%$ relative to normal age predicted values. ${ }^{18}$

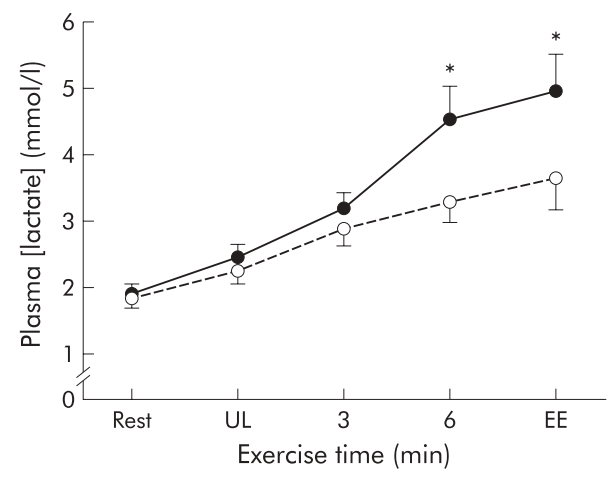

Figure 2 Plasma lactate response during continuous (closed circles) and intermittent exercise (open circles). Error bars denote SE. UL, unloaded cycling; $E E$, end exercise. The rest intervals were eliminated from the IE trend to equalise the time line between exercise modes. ${ }^{*} p<0.05$, continuous exercise $v$ intermittent exercise. 


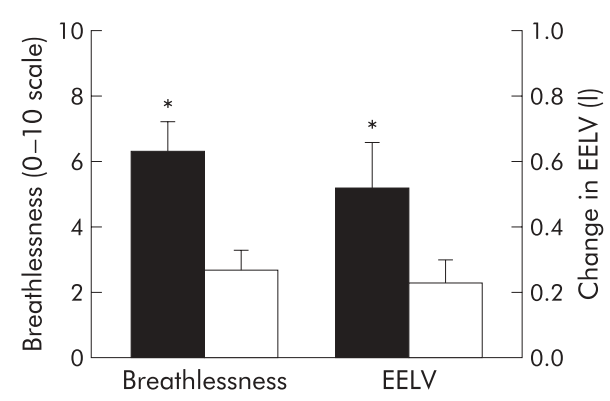

Figure 3 Change from rest to end of exercise in ratings of perceived breathlessness and end expiratory lung volume (EELV) during continuous (closed bars) and intermittent exercise (open bars). Error bars denote SE. ${ }^{*} \mathrm{p}<0.05$, continuous exercise $v$ intermittent exercise.

Figure 1 shows the cardiopulmonary responses measured during CE and IE. A significant mode by time interaction was observed for $\dot{\mathrm{V}}_{2}(\mathrm{~F}=41.87, \mathrm{p}<0.001), \dot{\mathrm{V}} \mathrm{CO}_{2}(\mathrm{~F}=55.30$, $\mathrm{p}<0.001), \dot{\mathrm{V}} \mathrm{E}(\mathrm{F}=21.57, \mathrm{p}<0.001)$ and heart rate $(\mathrm{F}=24.43$, $\mathrm{p}<0.001)$. For both modes of exercise $\dot{\mathrm{V}}_{2}, \dot{\mathrm{V}} \mathrm{CO}_{2}$ and $\dot{\mathrm{V}} \mathrm{E}$ increased significantly from the unloaded to loaded exercise transition (measured 1 minute after the workload was applied). These variables then remained relatively unchanged throughout the duration of IE, but continued to increase significantly up to 6 minutes of CE. During CE the end exercise values for $\dot{\mathrm{V}} \mathrm{O}_{2}, \dot{\mathrm{V}} \mathrm{CO}_{2}$ and $\dot{\mathrm{V}} \mathrm{E}$ were not significantly different from the 6 minute values. There was a significant increase in heart rate from unloaded cycling to the end of exercise in both CE and IE (fig ID). However, the increase in heart rate between consecutive time points was only significant from 3 minutes of $\mathrm{CE}$ onwards and between 6 minutes and the end of exercise during IE.

Continuous exercise resulted in significantly higher values for $\dot{\mathrm{V}}_{2}, \dot{\mathrm{V}} \mathrm{CO}_{2}, \dot{\mathrm{V}} \mathrm{E}$ and heart rate from 3 minutes of exercise onwards in comparison to IE. At the end of exercise the mean differences between CE and IE were: $\dot{\mathrm{V}}_{2} 0.25 \mathrm{l} / \mathrm{min}$ (95\% CI 0.15 to 0.34 ); $\dot{\mathrm{V}} \mathrm{CO}_{2} 0.34 \mathrm{l} / \mathrm{min}$ (95\% CI 0.23 to 0.45 ); $\dot{\mathrm{V}} \mathrm{E} 9.14 \mathrm{l} /$ min (95\% CI 4.94 to 13.30$)$; heart rate 15 beats $/ \mathrm{min}$ (95\% CI 7 to 21$)$. The predicted $\dot{\mathrm{V}}_{2}(0.85(0.09) \mathrm{l} / \mathrm{min})$ using $\tau$ values calculated from the individual CE tests was not significantly different from the values measured during IE (0.82 (0.07) l/ min). However, experimentally "speeding" on-transient kinetics through the use of a $\tau$ value derived from healthy older individuals resulted in predicted $\dot{\mathrm{V}}_{2}$ values $(0.97$ (0.10) $\mathrm{l} / \mathrm{min}$ ) that were significantly greater than the values measured in our subjects.

A significant mode by time interaction was also observed for plasma lactate concentrations $(\mathrm{F}=8.31, \mathrm{p}<0.001)$. Plasma lactate increased from rest through to the end of exercise during both IE and CE (fig 2). While plasma lactate concentrations during $\mathrm{CE}$ were systematically greater than during IE, the differences between exercise modes were only significant at 6 minutes and onwards. The increase in plasma lactate concentrations from rest to the end of exercise was significantly greater during CE (3.1 (0.7) $\mathrm{mmol} / \mathrm{l})$ than during IE (1.8 (0.4) mmol/l). The mean difference between exercise modes for plasma lactate concentration at end exercise was $1.7 \mathrm{mmol} / \mathrm{l}$ (95\% CI 0.8 to 2.5 ).

The subjects were able to exercise longer and to complete a greater amount of work during IE $(29.2$ (0.8) min; 70.5 (10.2) kJ) than during CE (11.1 (2.4) min; 30.5 (7.4) kJ). While eight of the 10 subjects were able to perform 60 minutes of IE (which amounts to 30 minutes of exercise), none was able to complete 30 minutes of CE.

Figure 3 shows the change from baseline to the end of exercise in EELV and breathlessness during IE and CE. The change in the degree of breathlessness from rest to the end of

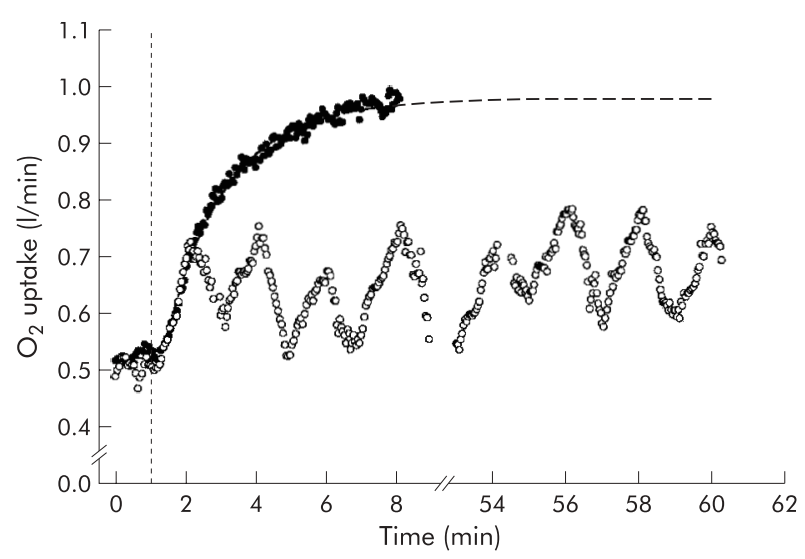

Figure 4 Oxygen uptake responses to a single bout of continuous (closed circles) and intermittent exercise (open circles) in a representative subject. The data represent the middle five of seven breath average responses. Each exercise bout was preceded by 3 minutes of unloaded cycling, but only the final minute of unloaded cycling is shown. The vertical dotted line indicates application of the predetermined workload. For clarity, only transitions early and late in the intermittent bout are depicted. A line of best fit (dashed line) was applied to the continuous exercise data, projecting to its asymptotic value and extrapolated through to the end of the intermittent bout.

CE (5.60 (0.87); $95 \%$ CI 3.60 to 7.60 ) was significantly greater than that observed during IE (2.07 (0.54); 95\% CI 0.84 to 3.29). Similarly, EELV (a measure of DH) increased to a greater extent during CE (0.52 (0.13) l; $95 \%$ CI 0.19 to 0.85$)$ than during IE $(0.23(0.07)$ l; $95 \%$ CI 0.07 to 0.39$)$. There was a significant positive correlation between the degree of breathlessness measured at the end of exercise and the change in EELV for both CE $(r=0.61, \mathrm{p}<0.05)$ and IE $(r=0.67, \mathrm{p}<0.05)$.

\section{DISCUSSION}

In this study patients with COPD were able to complete a greater total amount of work during IE than during CE. Since the majority of the subjects attained the 60 minute time limit during IE but none achieved the 30 minute CE limit, there was potential for an even greater amount of work to be completed during IE. Intermittent exercise was associated with a lower measured physiological response, with $\dot{\mathrm{V}}_{2}$, $\dot{\mathrm{V}} \mathrm{CO}_{2}, \dot{\mathrm{V}} \mathrm{E}$, heart rate, and plasma lactate concentrations all systematically lower than in CE. Moreover, subjects had a lower level of dynamic hyperinflation during IE than during $\mathrm{CE}$, as assessed by the change in EELV, and this may in turn have contributed to their lower sensation of breathlessness. The results of the present study indicate that IE is better tolerated than CE in patients with COPD because of improved ventilatory mechanics as well as reduced metabolic and cardiovascular perturbations.

As previously discussed, the amplitude of the $\dot{\mathrm{V}}_{2}$ response at a given exercise intensity during IE is determined primarily by the duration of the exercise period. ${ }^{7}$ The 1 minute intervals used during IE in the present study would therefore result in lower $\dot{\mathrm{V}}_{2}$ as well as $\dot{\mathrm{V}}_{\mathrm{CO}_{2}}$, $\dot{\mathrm{V}} \mathrm{E}$ and heart rate values, given that these variables are tightly coupled to metabolism within the active muscle during exercise. When exponential models were applied to the $\dot{\mathrm{V}}_{2}$ data measured during the CE bouts, the predicted $\dot{\mathrm{VO}}_{2}$ values at the end of 1 minute of exercise were not significantly different from the $\dot{\mathrm{V}}_{2}$ values obtained during the exercise intervals of the IE bouts (see online data supplement at www.thoraxjnl.com/supplemental). Moreover, the measured $\dot{\mathrm{V}}_{2}$ values were not significantly different between exercise modes during the first minute of exercise following the application of the predetermined workload 
(fig 1). Figure 4 clearly illustrates the similar time course for the increase in $\dot{\mathrm{V}}_{2}$ during the first minute of exercise for both modes of exercise in a representative subject. The responses then diverge as the rest intervals are introduced into the IE bout. The peak exercise $\dot{\mathrm{V}}_{2}$ response for the remainder of IE remains similar to the value measured at the end of the first exercise interval. Thus, the lower $\dot{\mathrm{V}}_{2}$ value observed during IE than during CE appears to be due to the delayed kinetic response typical of a step change in activity level (for example, from rest to exercise).

One aspect of cardiorespiratory kinetics that may also have an impact on the amplitude of the gas exchange responses is the "speed" with which these variables respond to a change in exercise intensity. The slowing of on-transient $\dot{\mathrm{V}}_{2}$ kinetics in patients with COPD relative to healthy control subjects ${ }^{12}{ }^{13}$ may result in IE values below those expected for a given external workload for an age matched healthy subject. When $\dot{\mathrm{V}}_{2}$ was predicted using a $\tau$ value derived from healthy older individuals, ${ }^{16}$ the estimated value was significantly greater than that measured during IE (see online data supplement at www.thoraxjournal.com/supplemental). The dependence of $\dot{\mathrm{V}} \mathrm{O}_{2}$ on the $\tau$ value during the on-transient phase suggests that the lower $\dot{\mathrm{V}}_{2}$ values observed during IE compared with CE may also reflect a slower than normal on-transient response in patients with COPD than in healthy subjects.

The slower $\dot{\mathrm{V}}_{2}$ kinetics in individuals with COPD would result in a greater reliance on phosphocreatine stores and anaerobic glycolysis at the onset of exercise or during a transition from lower to higher exercise intensities in these individuals. ${ }^{12}$ Consequently, an earlier or relatively greater efflux of lactate into the blood may occur. Previous studies have reported an "excessive" lactic acidosis relative to $\dot{\mathrm{V}}_{2}$ in patients with COPD. ${ }^{19} 20$ During IE it has been shown that lactate production occurs primarily during the exercise period $^{21}$ while the rest period is associated with net lactate removal. ${ }^{22}$ Thus, despite the possibility of a greater reliance on anaerobic metabolism to fuel the energetic demands for IE, the short exercise intervals interspersed with rest periods resulted in systematically lower plasma lactate values during IE. The greater plasma lactate response associated with CE than with IE may also have contributed, in part, to the higher $\dot{V}$ E observed during CE. The buffering of hydrogen ions linked with lactic acid production ultimately results in the formation of "non-metabolic" $\mathrm{CO}_{2} \cdot{ }^{23}$ Both an increase in hydrogen ion concentration and excess non-metabolic $\mathrm{CO}_{2}$ production act to stimulate ventilation. ${ }^{24}$

Since exertional dyspnoea can be a major contributing factor to exercise limitation in patients with COPD, we decided to examine ventilatory mechanics during CE and IE. In many individuals with COPD the increasing ventilatory demand during exercise results in the progressive trapping of air due to incomplete lung emptying. ${ }^{2}$ The accompanying increase in EELV is termed dynamic hyperinflation. This increase in EELV during exercise, compounded by an already increased resting functional residual capacity (table 1), curtails the ability of the COPD patient to expand tidal volume in line with the greater ventilatory demand. ${ }^{25}$ An increase in $\dot{V} E$ must then be mediated primarily by an increase in breathing frequency, which reduces expiratory flow time and further exacerbates the degree of dynamic hyperinflation. Dynamic hyperinflation has been associated with an increase in dyspnoea and may play an important role in the observed exercise intolerance in patients with COPD. ${ }^{25}{ }^{26}$ In the present study the degree of dynamic hyperinflation was significantly lower during IE than in CE. Moreover, EELV showed a significant positive correlation with perceived breathlessness. Given the dependence of dynamic hyperinflation on $\dot{V} \mathrm{E}$, it seems reasonable to suggest that the lower level of perceived breathlessness and dynamic hyperinflation observed during
IE was primarily a function of the lower Vंe during this mode of exercise. In addition, the rest intervals may have provided the subjects with the opportunity to "deflate" their lungs, allowing the lung volume to return to or approach passive FRC before the start of the next exercise bout. This would reduce the degree of dynamic hyperinflation achieved in the subsequent exercise bout.

The findings of the present study suggest that further examination of training intensity and volume, as well as exercise mode, could improve exercise prescription practices in pulmonary rehabilitation programmes. In particular, the use of IE training at intensities comparable to those used in CE protocols may result in similar levels of functional improvement. Recently, Morris et $a l^{27}$ and Vogiatzis et $a l^{4}$ compared CE and IE training in healthy older individuals and in patients with COPD, respectively. These studies showed that, when total work performed was equalised between the exercise modes, the benefits gained were independent of exercise mode as well as intensity. Furthermore, it is our opinion that IE training could possibly benefit individuals ranging in disease severity. For those individuals with severe airflow limitation, breathlessness can be a major psychological deterrent to the pursuit of an active lifestyle. ${ }^{14}$ The decreased perception of breathlessness associated with IE may improve adherence to training programmes and encourage individuals with lung disease to reverse the debilitating effects of a sedentary lifestyle. The greater options associated with IE protocol design (exercise to rest ratios as well as a greater range of tolerated intensities) could benefit those with mild disease. Even if lung mechanics were not a limiting factor, there is evidence that metabolic and morphological changes in the skeletal muscles of patients with COPD may result in a reduced exercise tolerance. ${ }^{1}$ In the present study the lower measured levels of plasma lactate during IE compared with CE suggest either a lower degree of metabolic perturbation during IE or greater lactate clearance (during the rest intervals) with better maintenance of intramuscular as well as blood homeostasis. This would be of benefit to individuals whose exercise tolerance is limited by peripheral factors.

In summary, we have shown that individuals with moderate COPD were able to complete a greater amount of work during IE than during CE performed at $70 \%$ of peak power. Measures of gas exchange, plasma lactate, dynamic hyperinflation, and perceived breathlessness were significantly lower during IE than during CE. The greater amount of work performed and lower measured physiological responses achieved with IE may allow for greater peripheral training adaptations in individuals with more limited lung function. The results suggest that IE may be superior to CE as a mode of training for patients with COPD.

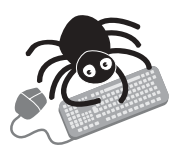

Further details are given in the online supplement available at www.thoraxjnl.com/supplemental

\section{Authors' affiliations}

S Sabapathy, R A Kingsley, D A Schneider, L Adams, N R Morris, School of Physiotherapy and Exercise Science, Gold Coast Campus, Griffith University, Queensland, Australia

Supported by the Breathlessness Research Charitable Trust, UK

\section{REFERENCES}

1 American Thoracic Society/European Respiratory Society. Skeletal muscle dysfunction in chronic obstructive pulmonary disease. Am J Respir Crit Care Med 1999; 159:S1-40.

2 O'Donnell DE. Ventilatory limitations in chronic obstructive pulmonary disease. Med Sci Sports Exerc 2001;33:S647-55. 
3 Maltais $F$, LeBlanc $P$, Jobin J, et al. Intensity of training and physiologic adaptation in patients with chronic obstructive pulmonary disease. Am J Respir Crit Care Med 1997; 155:555-61.

4 Vogiatzis I, Nanas S, Roussos C. Interval training as an alternative modality to continuous exercise in patients with COPD. Eur Respir J 2002;20:12-9.

5 Coppoolse R, Schols AMWJ, Baarends EM, et al. Interval versus continuous training in patients with severe COPD: a randomized clinical trial. Eur Respir J 1999; 14:258-63.

6 Smodlaka VN, Adamovich DR. Reconditioning of emphysema patients. NY State J Med 1974;74:951-5.

7 Åstrand I, Åstrand P-O, Christensen EH, et al. Intermittent muscular work. Acta Physiol Scand 1960;48:448-53.

8 Morris N, Gass G, Thompson M, et al. Physiological responses to intermittent and continuous exercise at the same relative intensity in older men. Eur J Appl Physiol 2003;90:620-5.

9 Åstrand I, Åstrand P-O, Christensen EH, et al. Myohemoglobin as an oxygenstore in man. Acta Physiol Scand 1960:48:454-60.

10 Whipp BJ, Ward SA, Lamarra N, et al. Parameters of ventilatory and gas exchange dynamics during exercise. J Appl Physiol 1982;52:1506-13.

11 Barstow TJ. Characterisation of $\dot{\mathrm{V}}_{2}$ kinetics during heavy exercise. Med Sci Sports Exerc 1994;26:1327-34.

12 Palange P, Galassetti $\mathrm{P}$, Mannix ET, et al. Oxygen effect on $\mathrm{O}_{2}$ deficit and $\mathrm{V}_{2}$ kinetics during exercise in obstructive pulmonary disease. J Appl Physiol 1995;78:2228-34.

13 Nery LE, Wasserman K, Andrews JD, et al. Ventilatory and gas exchange kinetics during exercise in chronic airways obstruction. J Appl Physiol 1982;53:1594-602

14 Kaplan RM, Eakin EG, Ries AL. Psychosocial issues in the rehabilitation of patients with chronic obstructive pulmonary disease. In: Casaburi R, Petty TL, eds. Principles and practice of pulmonary rehabilitation. Philadelphia: W B Saunders, 1993:351-65

15 Pauwels RA, Buist AS, Calverley PMA, et al. Global strategy for the diagnosis, management, and prevention of chronic obstructive pulmonary disease.
NHLBI/WHO global initiative for chronic obstructive lung disease (GOLD) workshop summary. Am J Respir Crit Care Med 2001;163:1256-76.

16 Sabapathy S, Schneider DA, Comadira G, et al. Oxygen uptake kinetics during severe exercise: a comparison between young and older men. Respir Physiol Neurobiol 2004;139:203-13.

17 Lansing RW, Moosavi SH, Banzett RB. Measurement of dyspnea: word labeled visual analog scale vs. verbal ordinal scale. Respir Physiol Neurobiol 2003; 134:77-83

18 Jones NL, Makrides L, Hitchcock C, et al. Normal standards for an incremental progressive cycle ergometer test. Am Rev Respir Dis 1985;131:700-8.

19 Maltais F, Simard A-A, Simard C, et al. Oxidative capacity of the skeletal muscle and lactic acid kinetics during exercise in normal subjects and in patients with COPD. Am J Respir Crit Care Med 1996;153:288-93.

20 Casaburi R, Patessio A, loli F, et al. Reductions in exercise lactic acidosis and ventilation as a result of exercise training in patients with chronic obstructive lung disease. Am Rev Respir Dis 1991;143:9-18.

21 Saltin B, Essén B. Muscle glycogen, lactate, ATP and CP during intermittent exercise. In: Pernow B, Saltin B, eds. Muscle metabolism during exercise. New York: Plenum Press, 1971:419-24.

22 Essén B. Studies on the regulation of metabolism in skeletal muscle using intermittent exercise as an experimental model. Acta Physiol Scand 1978;454:S3-32.

23 Wasserman K, Hansen JE, Sue DY, et al. Principles of exercise testing and interpretation, 3rd ed. Baltimore: Lippincott, Williams \& Wilkins, 1999.

24 Whipp BJ. The control of exercise hyperpnea. In: Hornbein T, ed. Regulation of breathing. New York: Marcel Dekker, 1981:1069-139.

25 Babb TG, Viggiano R, Hurley B, et al. Effect of mild-to-moderate airflow limitation on exercise capacity. J Appl Physiol 1991;70:223-30.

26 O'Donnell DE, Revill SM, Webb KA. Dynamic hyperinflation and exercise intolerance in chronic obstructive pulmonary disease. Am J Respir Crit Care Med 2001; 164:770-7.

27 Morris N, Gass G, Thompson M, et al. Rate and amplitude of adaptation to intermittent and continuous exercise in older men. Med Sci Sports Exerc 2002;34:471-7.

\section{LUNG ALERT}

\section{Adjunct prednisolone therapy does not improve survival in HIV-associated tuberculous pleurisy}

$\Delta$ Elliott AM, Luzze H, Quigley MA, et al. A randomized, double-blind, placebo-controlled trial of the use of prednisolone as an adjunct to treatment in HIV-1-associated pleural tuberculosis. J Infect Dis 2004; 190:869-78

G lucocorticoids reduce excessive inflammation in pleural tuberculosis and have been shown to hasten resolution in HIV negative patients. Active tuberculosis can speed progression of HIV infection since HIV replicates more rapidly in activated lymphocytes. Given that prednisolone therapy is associated with reduced immune activation in HIV positive subjects, the authors hypothesised that adjunct therapy with prednisolone in HIV positive patients with pleural tuberculosis would decrease viral replication and improve survival.

In this study, 197 Ugandan HIV infected patients with pleural tuberculosis were randomised to receive treatment for 8 weeks with either a reducing dose of prednisolone or placebo, together with 6 months of treatment with a four-drug antituberculous regime. The median follow up periods were 1.65 and 1.48 years in the prednisolone and placebo groups, respectively. Prednisolone had no effect on either survival or HIV viral load. The mortality rate ratio for prednisolone compared with placebo, after adjusting for confounding factors, was 0.99 (95\% CI 0.62 to $1.56, p=0.95)$. Use of prednisolone was associated with significantly more rapid improvements in anorexia, weight loss, cough, and radiological resolution of pleural effusion. However, use of prednisolone was also associated with a significantly increased risk of adverse effects $(9 \%)$ compared with placebo $(2 \% ; \mathrm{p}=0.03)$, and a significantly higher incidence of Kaposi's sarcoma (prednisolone 4.2 cases/100 personyears; placebo 0 cases/100 person-years, $\mathrm{p}=0.02$ ).

In light of the lack of survival benefit and the increased risk of Kaposi's sarcoma, the authors recommend that prednisolone should not be used in HIV associated tuberculous pleurisy. 